
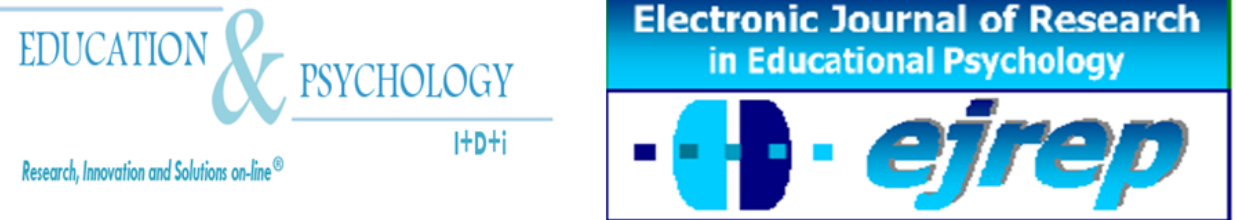

Editorial EOS

\title{
El proceso de toma de decisiones profesionales a través del coaching
}

\author{
Manuel Álvarez González y Meritxell Obiols Soler
}

${ }^{1}$ Departamento MIDE. Universidad de Barcelona

\section{España}

alvarez.m@,ub.edu

Dirección Postal: Universidad de Barcelona. Departamento MIDE. Campus Mundet. Passeig de la Vall d'Hebron, 171. 08035 Barcelona. España

(C) Education \& Psychology I+D+i and Editorial EOS (Spain) 


\title{
Resumen
}

En este trabajo se afronta, de una forma comprensiva, el proceso de la toma de decisiones en la realidad del coaching. Para ello, se señalan aquellos aspectos a tener en cuenta en una toma de decisiones de calidad. Se hace una aproximación a los principales enfoques para el desarrollo de este proceso. Igualmente se propone un modelo comprensivo para la toma de decisiones con sus dimensiones y descriptores. Termina este estudio con la presentación de algunas estrategias de coaching para el desarrollo de cada una de las dimensiones de este modelo.

Palabras clave: Coaching, toma de decisiones, modelos, dimensiones, estrategias.

Recibido: 04/11/08 Aceptación inicial: 07/11/08 Aceptación final: 14/01/09

\section{The process of making professional decisions through coaching}

\begin{abstract}
In this work one confronts, of a comprehensive form, the process of the decision making in the reality of coaching. For it, those aspects are indicated to consider in a decision making of quality. An approach to the main approaches for the development becomes of this process. Also one proposes a comprehensive model for the decision making with his dimensions and descriptors. This study with the presentation of some strategies of coaching for the development of each one of the dimensions of this model finishes.
\end{abstract}

Keywords: Coaching, decision making, models, dimensions, strategies.

Received: 04/11/08 Initial Acceptance: 07/11/08 Definitive Acceptance: 14/01/09 


\section{Introducción}

El mundo laboral se ve sometido a la continua toma de decisiones; de ahí que la nueva realidad profesional haya de contemplar, como un componente fundamental, la ayuda en los procesos de toma de decisiones profesionales. Éste es un proceso complejo donde interactúan diferentes factores o dimensiones no sólo de tipo cognitivo, sino de tipo emocional y social. Su desarrollo se ha concretado en diferentes apartados, que contemplan los aspectos más relevantes del tema que nos ocupa:

a) En primer lugar, se efectúa un breve análisis de lo que significa el coaching y su relación con los procesos de toma de decisiones. Este panorama reclama, entre otros aspectos, la necesidad de desarrollar en los colaboradores competencias para una adecuada toma de decisiones.

b) En segundo lugar, se clarifica el concepto de la toma de decisiones; se señalan los aspectos a destacar en dicho proceso, los estilos y las estrategias para tomar una decisión y los factores que influyen positivamente en la decisión, los que se asocian con la indecisión. Asimismo, se presentan aquellos componentes que se han de tener presentes en toda intervención de coaching en la toma de decisiones profesionales: 1) los enfoques teóricos que fundamentan dicha intervención (enfoques de toma de decisiones personales y enfoques de toma de decisiones profesionales); 2) un modelo comprensivo para la toma de decisiones profesional con sus tres dimensiones (cognitiva, emocional y social) y 3) algunas estrategias de coaching para la toma de decisiones profesional, teniendo en cuenta las tres dimensiones del modelo propuesto.

La finalidad de este trabajo es hacer reflexionar sobre el proceso decisorio, facilitando algunas orientaciones y recursos para la intervención del coach.

\section{¿Qué entendemos por coaching?}

El coaching participa de la concepción humanista según la cual el hombre es la medida de todas las cosas. La dignidad y el valor del individuo es insuperable y las necesidades humanas prevalecen sobre las cosas materiales. El coaching trabaja con la "autoconsciencia" y la necesidad de autorrealización del individuo, la consecución del pleno potencial, la autoexpresión, el logro y el crecimiento (Zeus y Skiffington, 2004). 
El coaching es "un proceso de entrenamiento personalizado y confidencial llevado a cabo por un asesor especializado o coach, que cubre el vacío existente entre lo que eres ahora y lo que deseas ser. Es una relación profesional con otra persona que aceptará sólo lo mejor de la misma y te aconsejará, guiará y estimulará para que vayas más allá de las limitaciones que te impones a ti mismo para que realices tu pleno potencial" (Miedaner, 2004: 23).

Cuando se trabaja con un coach, se producen una serie de cambios en la persona que lo recibe. Entre ellos: a) se toma todo más en serio; b) las decisiones se afrontan de forma inmediata, resultando más focalizadas y efectivas; c) se crea un ambiente en el que es más fácil obtener las metas propuestas y d) se obtienen mejores resultados de los que se hubieran podido alcanzar sin el coach.

Según Zeus y Skiffington (2004), el coaching es considerado un proceso en el que el coach y el coachee conciben el problema y la solución mediante el lenguaje. El coaching es consciente de que siempre existen formas alternativas de vislumbrar el mundo y que el coachee no se encuentra limitado por sus experiencias anteriores a la hora de adoptar nuevas y útiles interpretaciones de sí mismo y del problema.

El coaching viene centrándose históricamente en la mejora de determinada actuación de comportamiento. El coach eficaz observa el comportamiento de la persona y luego le da consejos e indicaciones de cómo mejorar en situaciones y contextos específicos. Ello implica promover el desarrollo de la capacidad del comportamiento de esa persona a través de una cuidadosa observación y de la correspondiente interacción (Dilts, 2004).

Los conceptos de elección y responsabilidad son cruciales para el coaching, cuya alianza no prosperará si el coachee no se compromete firmemente a cambiar su visión del mundo, sus creencias autolimitadoras o sus acciones autodestructivas. Según este enfoque, es nuestro modo de ver el mundo lo que determina nuestras acciones en todo momento (Zeus y Skiffington, 2004).

El coaching está relacionado tanto con el modo de hacer cosas como con lo que se hace. En gran medida, el coaching proporciona resultados debido a la relación de apoyo que se establece entre el coach y la otra persona, junto con los medios y el estilo de comunicación que se han utilizado. La persona toma conciencia de los hechos por sí misma, estimulada por 
un coach. Por supuesto, el objetivo fundamental del coaching es mejorar el desempeño, sin embargo, lo importante es tratar de averiguar la mejor manera de conseguirlo (Whitmore, 2005).

El coaching, "más que un entrenamiento, es entendido como una disciplina, un arte, un procedimiento, una técnica y también un estilo de liderazgo, gerenciamiento y conducción" (Wolk, 2004, pág. 23). Por su parte, Launer (2007) definió el coaching como arte de facilitar el desarrollo potencial de las personas y de los equipos para alcanzar objetivos coherentes y cambios profundos.

En definitiva, contratan un coach aquellos que quieren más, que desean crecer y hacer las cosas más fácilmente, como por ejemplo, tomar decisiones. Aquellos que llevan meses e incluso años, pensando en cosas que desearían hacer, tener o ser, pero que nunca se decidieron a empezar, o que siempre se pararon en el mismo punto y realmente quieren lograrlo y superar sus limitaciones. Un coach te puede ayudar a ser, a hacer, a obtener lo que buscas y tanto deseas.

\section{La nueva realidad del coaching y los procesos de toma de decisiones}

La nueva realidad del coaching se podría concretar en los siguientes aspectos o características:

- La formación integral, comprensiva y diversificada del cliente, que le prepare para el acceso a los diferentes retos profesionales. Esto va a requerir, entre otros aspectos, el desarrollo de competencias para asumir los procesos de toma de decisiones.

- Esta formación ha de resaltar, además de la dimensión cognitiva, las dimensiones afectivo-emocional y social.

- Con un mayor grado de opcionalidad que van a implicar al coachee el tener que hacer elecciones adecuadas y constantes.

- Supone una mayor libertad y responsabilidad. El coachee se enfrenta a constantes situaciones de decisión que ha de superar de forma correcta.

- Mayor diversidad de los clientes en cuanto a preparación, motivación, intereses, valores, expectativas, etc. 
- Proceso de coaching caracterizado por la necesidad de una adecuada exploración del yo (conocimiento de sí mismo) y del entorno laboral.

Uno de los factores que determinan la calidad de un proceso de coaching es afrontar la preparación para los procesos de toma de decisiones de tipo personal y socio-laboral que el cliente ha de realizar a lo largo de su trayectoria profesional. Como se verá en este trabajo, hay que preparar al cliente para que pueda asumir, con las mayores garantías de éxito, este proceso, no sólo desde el punto de vista competencial (saber tomar la decisión), sino también afectivo-emocional (tener en cuenta aquellos aspectos emocionales que entran en juego) y social (contar con las personas que nos rodean).

\section{La toma de decisiones. Aspectos a tener en cuenta.}

Por toma de decisiones se entiende todo un proceso o actividad de procesamiento de la información necesaria, que permite llegar a un fin satisfactorio. Debe entenderse como un proceso continuo de carácter cognitivo, emocional y social. Este proceso requiere de una constante revisión en función de las nuevas informaciones que el sujeto va adquiriendo a lo largo de su vida, además de una implicación por parte del propio interesado (saber interiorizar el proceso y querer afrontarlo). La persona ha de sentirse identificada con cada una de sus decisiones. La decisión es la elección que sigue a la deliberación consciente y reflexiva.

\section{Aspectos a destacar en los procesos de toma de decisiones.}

Una vez definido el concepto de toma de decisiones, se está en situación de destacar aquellos aspectos que le son comunes (Álvarez González, 2004; Álvarez González y Rodríguez Moreno, 2006):

- Se ha de decidir en un tiempo y/o momento determinado. Una decisión la hace fácil o difícil el tiempo de que se dispone para llevarla a cabo y el momento en que se produce, que puede ser adecuado o no.

- Constituye una situación de cambio y/o ruptura con lo anterior. Toda decisión supone un cambio que puede ser incluso traumatizante, si no se prepara a la persona. Este cambio o ruptura implica salir de un contexto y entrar en otro, que, en la mayoría de los casos, no 
se parece al anterior. Este proceso se ha de preparar previamente para que la persona sepa lo que se va a encontrar y cómo adaptarse a esa nueva situación.

- Se plantean diferentes alternativas/trayectorias/propuestas. Se han de poner sobre la mesa todas las alternativas, propuesta, trayectorias en el caso de que se quiera realizar una correcta toma de decisiones.

- Se requiere de una plena implicación por parte del interesado. La persona que tiene que tomar una decisión, resolver un problema o bien llevar acabo una transición es el sujeto activo del proceso. Él y sólo él es el que tiene que asumir dicho proceso. El resto de agentes implicados (personas próximas) han de acompañarle y estar a su lado cuando necesite ayuda.

- Suponen un proceso de aprendizaje permanente a lo largo de la vida. La toma de decisiones constituye un proceso de aprendizaje. Como estos procesos son continuos a lo largo de la vida del individuo requieren un aprendizaje permanente.

- Implican una adaptación personal y social a la nueva situación. Estos procesos de cambio y, a veces de ruptura, suponen no sólo todo un proceso de adaptación personal, sino también contextual a la nueva realidad.

- Comportan momentos críticos antes, durante y después. La complejidad de los procesos de toma de decisiones, de resoluciones requieren de una preparación (antes), de una puesta en ejecución (durante) y de un seguimiento (después). Se ha de preparar el proceso, se ha de acompañar a la persona en la ejecución y se ha de hacer un seguimiento para comprobar si la decisión tomada ha sido la adecuada.

- Se ponen en juego tres dimensiones: la cognitiva, la emocional y la social. Para desarrollar, de forma plena, este proceso se ha de tener en cuenta la dimensión cognitiva (competencias para saber tomar la decisión, resolver un problema o realizar una transición), la dimensión emocional (competencias afectivo-emocionales) y la dimensión social (tener en cuenta a los que nos rodean).

- Se han de describir los sentimientos y emociones y evitar los que sean negativos. Se ha de tomar conciencia de cómo las emociones desempeñan un papel importante en los procesos de toma de decisiones. Es necesario conocer las propias emociones y las de los demás para luego controlarlas. Estos procesos tienen efectos importantes sobre los estados emocionales y se han de evitar en todo momento las emociones negativas (ansiedad, temor, ira, vergüenza, miedo, etc.). 
- Se ha de evitar tanto la impulsividad como la inhibición (autocontrol). En estos procesos el sujeto no puede ser impulsivo, ni inhibirse, sino que debe ser reflexivo, si quiere abordar de forma adecuada la toma de decisiones. Se ha de tener un adecuado autocontrol durante todo el proceso (asertividad).

\section{Estilos y estrategias para tomar una decisión}

Diferentes autores (Álvarez González, 2004; Álvarez González y Rodríguez Moreno, 2006; Blustein, 1987; Corominas, 1999; Harren, 1979; Jepsen, 1974; Johnson, 1978; Krumboltz, 1979; Niles, et al. 1997), entre otros, han señalado que en el proceso de toma de decisiones se pueden adoptar diferentes estilos y estrategias de decisión. Estos se podrían agrupar en:

- Activos: Se implican desde el primer momento en el proceso y son conscientes de su papel. Sienten la necesidad de tener que asumir las decisiones y tomar la iniciativa desde el principio. Se ven protagonistas y manifiestan una fuerte voluntad por implicarse en dicho proceso.

- Pasivos: Les cuesta más afrontar la elección. Se ven influenciados por los que le rodean a la hora de tomar sus propias decisiones, quieren satisfacer a los demás y se sienten seguros si otras personas respaldan su decisión. En definitiva quieren que los demás le saquen "las castañas del fuego" y sean los que lleven la "voz cantante".

- Lógicos: Manifiestan a lo largo de todo el proceso un propósito definido por afrontar sus propias decisiones y por querer resolver sus problemas con la ayuda de sí mismo y de los demás. Valoran los pros y contras de forma objetiva, razonada y sin precipitación. Ven este proceso como algo que forma parte de su proyecto personal y profesional.

- Impulsivos y emocionales: Los sujetos se guían más por lo emocional que por lo cognitivo y suelen mostrarse un tanto impulsivos en sus acciones. No maduran lo suficiente los diferentes pasos que han de seguir antes de tomar la decisión definitiva. Se mueven por impulsos emocionales y no tanto por razonamientos lógicos y racionales. La toma de decisiones depende, en gran medida, de su estado de ánimo. 


\section{Factores que influyen en la toma de decisiones}

La toma de decisiones es un proceso complejo en el que intervienen una serie de factores que determinan el éxito del proceso. Diferentes autores (Álvarez González, 2004; Gómez, 1995; Ma y Yeh, 2005; Osipow, 1983; Rivas, 1988) destacan los siguientes factores:

De tipo personal: La autoestima y autoconfianza, los valores, la personalidad, las aptitudes, las experiencias, la conciencia de las emociones y la regulación emocional.

De tipo motivacional: Las preferencias, las expectativas, las aspiraciones, los intereses y la satisfacción.

De tipo profesional: Salidas laborales, prestigio social, seguridad, salario, entre otros.

De tipo ambiental: Posibilidades del entorno, la situación económica, el apoyo y soporte familiar.

En definitiva, los factores que determinan la toma de decisiones son:

- La realidad que envuelve al sujeto que ha de tomar la decisión

- El conocimiento que ha de tener de sí mismo y de los demás

- La información suficiente que debe tener sobre el problema a decidir

- Los aspectos afectivo-emocionales que ha de tener presentes y que pueden ser favorecedores o no de la decisión.

\section{Factores que se asocian con la indecisión}

Una vez que se han señalado los factores que favorecen la decisión, se podrían igualmente destacar algunos de aquellos factores que se asocian con la indecisión del cliente. A partir de los estudios consultados (Álvarez González, 2005; Bergeron y Romano, 1994; Callanad y Grenhauss, 1990; Gaffner y Hazler, 2002; Gómez, 1995; Kraus y Hugher, 1999) los factores que se asocian con dicha indecisión son los siguientes:

- Ansiedad ante la elección. Un nivel de ansiedad alto puede bloquear el proceso de la toma de decisiones y provocar que el sujeto no actúe con objetividad, porque su estado emocional no es el adecuado. 
- Dudas sobre las propias posibilidades. Una persona que no conoce sus puntos fuertes o débiles, sus fortalezas y amenazas, no se encuentra en buena disposición para tomar decisiones profesionales de cierta trascendencia.

- Falta de información sobre sí mismo. Uno de los aspectos básicos a tener en cuenta en cualquier toma de decisiones es el conocimiento de sí mismo. Cuanto mayor sea el conocimiento de sí mismo en mejores condiciones estará para asumir su propio proceso de toma de decisiones.

- Dificultades para analizar alternativas. Hay personas que tienen dificultades para analizar y valorar las diferentes alternativas que se han de barajar en un adecuado proceso de toma de decisiones.

- Excesiva dependencia. Un sujeto que es excesivamente dependiente de lo que le rodea, que está constantemente pidiendo ayuda a todas las personas próximas a él, tiene serias dificultades para afrontar su propio proceso de toma de decisiones.

- Multiplicidad de intereses y de valores. Un sujeto que tiene poco cristalizados sus intereses (son dispersos y diversificados) y poco clarificados sus valores no está en la situación adecuada para llevar a cabo una toma de decisiones profesional.

- Falta de autoconfianza en la decisión tomada. Una vez tomada una decisión no se ha de estar constantemente reconsiderando la situación. Se ha de dejar pasar un tiempo antes de plantear una posible revisión de la decisión tomada.

\section{Enfoques teóricos de la toma de decisiones personal y profesional}

La intervención para la toma de decisiones requiere de unos planteamientos teóricos que van a fundamentar dicho proceso. Estos enfoques se pueden agrupar en torno a dos tipos: los referidos a los problemas personales (Modelo IDEAL de J. D. Bransford y B. S. Stein, 1993) y los centrados en la elección profesional. Y éstos a su vez se podrían agrupar en dos modelos: modelos descriptivos que explican las diferentes maneras cómo las personas toman decisiones, considerándolo como un fenómeno natural (Modelos de Tiedeman y O’Hara, 1963; Hilton, 1962; ; Pelletier y Cols, 1991; Super, 1977) y los modelos presciptivos que pretenden capacitar en la toma de decisiones, enseñando los factores que intervienen y las estrategias a utilizar en todo el proceso, con el fin de ayudar a tomar decisiones acertadas (Modelo de Gelatt, 1962, 1977; Holland, 1973, 1985; Krumboltz, 1979; Krumboltz y Hamel, 1977, entre otros). Estos enfoques se centran exclusivamente en la dimensión cognitiva o 
competencial para enseñar a tomar decisiones, pero olvidan las dimensiones afectivoemocional y social. Dentro de los modelos existentes destaca el modelo DECIDES de Krumbolt, que a continuación se presenta y que ha fundamentado a la mayoría de los programas de toma de decisiones publicados. Éste consta de las siguientes fases:

1. Definir bien la situación problemática. Explicitar lo que se desea y el tiempo límite para tomar la decisión.

2. Establecer un plan de acción. En esta fase se han de describir las acciones necesarias para tomar la decisión; se ha de planificar cuándo se hará cada actividad y se ha de estimar el tiempo que se dedicará a cada etapa o fase.

3. Clarificar valores. Clarificar los valores y considerar los beneficios.

4. Identificar alternativas. Barajar las diferentes opciones y alternativas.

5. Descubrir resultados posibles. Comparar las diferentes opciones.

6. Eliminar alternativas. Descartar aquellas alternativas que no responden a las necesidades planteadas.

7. Empezar la acción. Llevar a cabo la opción elegida.

Se ha podido confirmar en otro estudio (Álvarez González, 1991) la aplicabilidad de este modelo. El sujeto puede aprender a tomar decisiones de una forma racional a través de un entrenamiento de simulación que permite incidir en aquellas estrategias racionales de toma de decisiones. Igualmente, a partir de este modelo, se han diseñado y elaborado programas específicos de toma de decisiones (Benavent, 2002, 2006; Coronado, 2003; Frago y Álvarez Rojo, 2006, Rodríguez Moreno, Dorio y Morey, 1994, entre otros), concretamente en Álvarez González $(1995 ; 2004 ; 2006)$ se presentan una variedad de programas de toma de decisiones.

A nivel emocional se ha de destacar el modelo de Inteligencia emocional de J. D. Mayer., D. Caruso y P. Salovey (1999) para la toma de decisiones (Figura 1). 


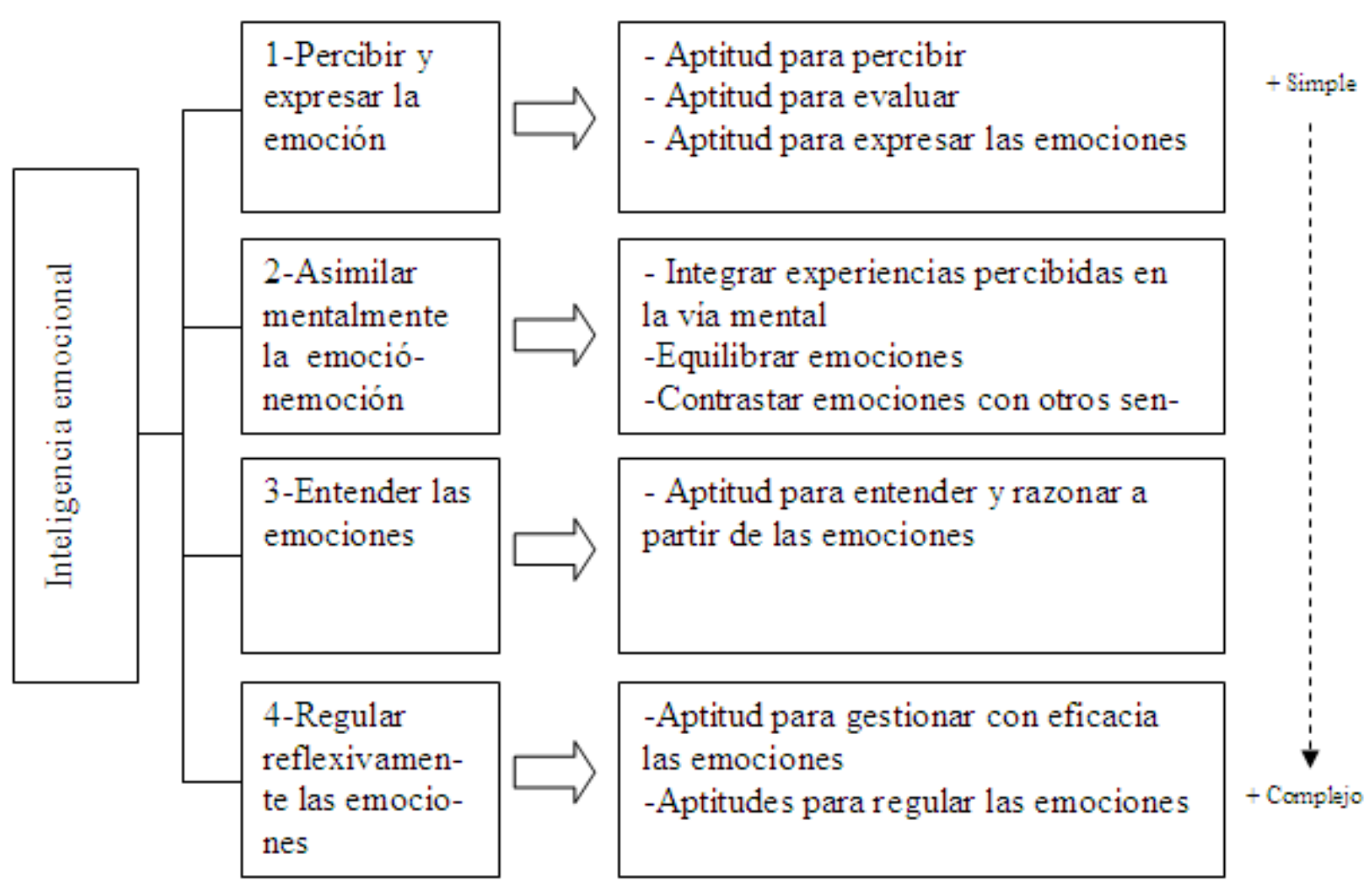

Figura 1. Modelo de Inteligencia emocional de Mayer, Caruso y Salovey (1999)

Este modelo se compone de cuatro fases que se desarrollan a lo largo de un contínuum que va desde el nivel de elaboración emocional más simple al nivel más complejo:

a) El nivel básico, percibir la emoción, supone la actitud para percibir, estimar y expresar las emociones

b) Un segundo nivel más complejo, donde tiene lugar la asimilación mental de las emociones. Se trata de poner bajo control cognitivo las emociones para poder asumir adecuadamente el proceso de toma de decisiones

c) El tercer nivel se basa en la comprensión de las emociones que exige una especial habilidad para comprender y razonar desde las emociones

d) Un cuarto y último nivel donde se concreta la gestión de las emociones, que es la capacidad de regular las propias emociones de una manera eficaz en el proceso de la toma de decisiones. 


\section{Modelo comprensivo para la toma de decisiones profesional}

Álvarez González (2005) y Álvarez González y Rodríguez Moreno (2006) nos presentan un modelo inédito para la toma de decisiones profesional, donde se contemplan las dimensiones cognitiva, emocional y social (Figura 2).

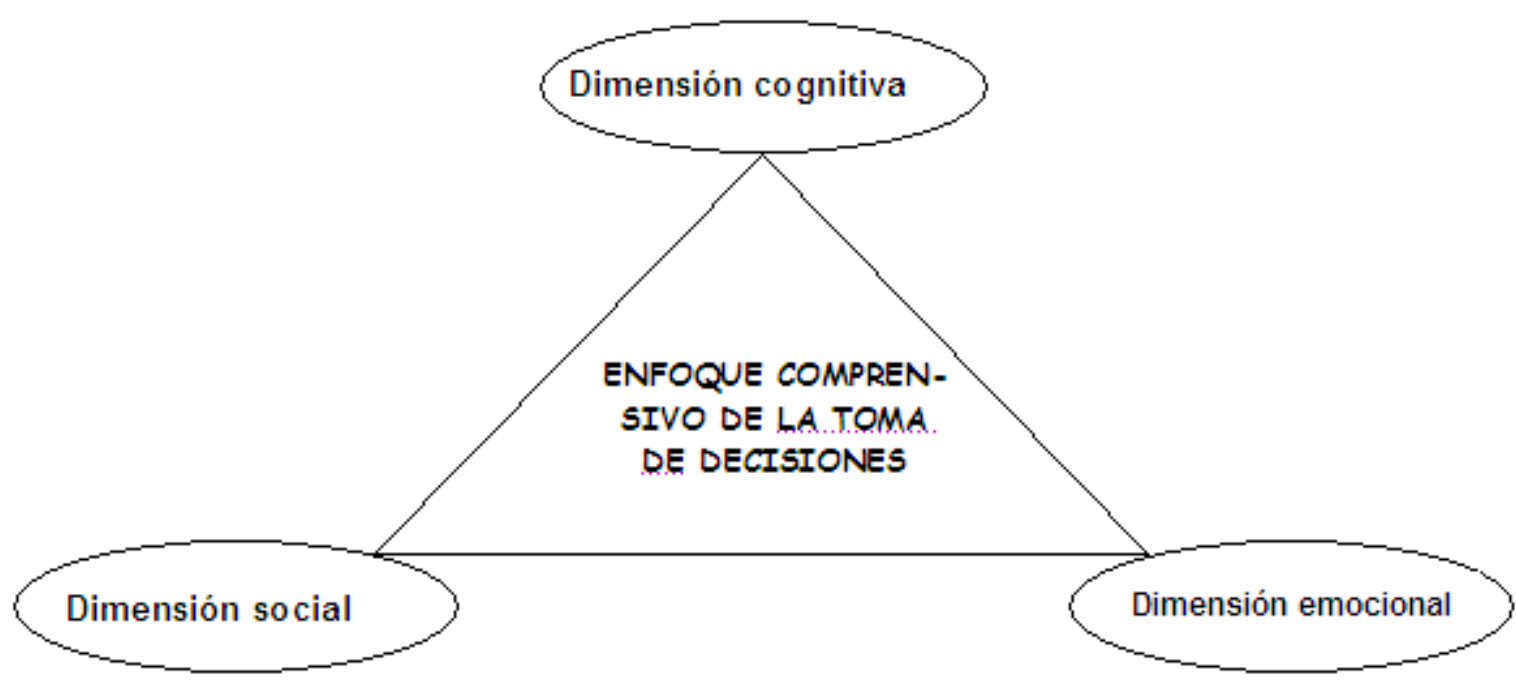

Figura 2. Enfoque comprensivo de la toma de decisiones de Álvarez González (2004) y Álvarez González y Rodríguez Moreno (2006)

A continuación se describen cada una de las dimensiones con sus diferentes factores y variables:

\section{Dimensión cognitiva}

Esta dimensión estudia cómo se podrían desarrollar en las personas una serie de competencias cognitivas que le preparen para saber tomar decisiones. Es decir, se trata de transmitir qué pasos se han de seguir para afrontar correctamente dicho proceso. Precisamente los diferentes enfoques teóricos desarrollan fundamentalmente esta dimensión.

En esta dimensión se debe incluir las siguientes áreas: 
- El conocimiento de sí mismo. La persona ha de conocer sus intereses a través de todo el proceso de coaching, sus valores, sus aptitudes, sus expectativas, sus experiencias, sus estilos cognitivos, etc.

- El conocimiento de los demás. Ha de desarrollar competencias interpersonales que le permitan conectar con aquellas personas que, en un momento determinado, le pueden ayudar en su proceso de decisión profesional.

- Información adecuada sobre el problema a decidir. Ha de barajar todas aquellas alternativas que es necesario tener en cuenta para tomar una decisión adecuada.

- Habilidad para evaluar y organizar la información relevante. Debe saber analizar de forma correcta y objetiva las diferentes alternativas que van a ser imprescindibles en la decisión.

- Habilidad o eficacia para resolver problemas. Tiene que estar en posesión de las competencias básicas para afrontar con éxito la toma de decisiones.

\section{Dimensión emocional}

Esta es una dimensión que tiene un peso muy importante a lo largo del coaching si se quiere asumir el proceso de toma de decisiones de forma comprensiva y plena. Es necesario tener en cuenta otros aspectos de carácter emocional que están presentes en la decisión y hacen que el individuo quiera o no tomar la decisión; el dominio de estos aspectos puede hacer más fácil la toma de decisiones. Estos aspectos son:

- La autoestima y la confianza en sí mismo. Una adecuada valoración de sí mismo e igualmente una suficiente y correcta confianza en sí mismo

- El tomar conciencia de las propias emociones y las de los demás

- El manejo y regulación de las propias emociones, evitando las emociones negativas (ansiedad, miedo, temor, tristeza, ira, vergüenza, estados depresivos, etc.)

- El control de la ansiedad ante la elección como un requisito básico e imprescindible

- La resolución de conflictos psicoemocionales. Supone una carencia de intereses, falta de confianza en sí mismo, falta de identidad personal y profesional, sentimientos de conflicto, miedo a tener éxito, miedo a fracasar, etc. 
La emoción a veces impide la decisión y de la indecisión surge el conflicto. Tomar conciencia de los factores emocionales en la toma de decisiones, en la resolución de problemas o en las transiciones contribuye a lograr un mejor autoconocimiento para tomar decisiones, resolver conflictos y hacer transiciones más apropiadas.

\section{Dimensión social}

El proceso de coaching debe favorecer la toma de conciencia de que en toda toma de decisiones se está implicando a los que nos rodean. De ahí la importancia de hacer partícipes de nuestra decisión a aquellas personas que están próximas a nosotros y forman parte de nuestras vidas (pareja, hijos/as, etc.).

En esta dimensión se desarrollan las competencias interpersonales y el apoyo de las personas que nos rodean. Los factores a tener en cuenta son:

- Apoyo del contexto. Las posibilidades que nos ofrece el entorno, el nivel cultural y clase social de los que nos rodean.

- Apoyo afectivo y respaldo familiar. La familia ha de dar el apoyo y la comprensión necesaria para hacerla más fácil y llevadera.

- Apoyo económico. Disponer del apoyo económico suficiente que no interfiera, sino que favorezca la decisión.

- Dominio de las habilidades sociales básicas. Saber escuchar, pedir disculpas, despedirse, etc.

- Respeto por los demás. Aceptar y apreciar las diferencias individuales y grupales y valorar los derechos de todas las personas.

- Comunicación receptiva (capacidad para atender a los demás) y comunicación expresiva (capacidad para expresar los propios pensamientos y sentimientos con claridad).

- Compartir emociones positivas y sinceras en los momentos de decisión.

- Comportamiento prosocial y cooperación. Mantener actitudes de amabilidad y respeto a los demás.

- Asertividad. Mantener un comportamiento equilibrado entre la agresividad y la pasividad, capacidad para defender y expresar los propios derechos, opiniones y sentimientos. 
Las decisiones adecuadas implican la integración de lo racional, lo emocional y social. Es difícil pensar en algún problema o elección profesional que no esté asociado con emociones y relaciones sociales. Se han de tomar en consideración las emociones y las habilidades interpersonales. Investigaciones recientes (Brown, George-Currant y Smith, 2003; Emmerling y Cherniss, 2003; Gaffner y Hazler, 2002; Mellers, Schwartz y Ritov, 1999) demuestran que en los procesos de toma de decisiones personales y profesionales están más presentes los factores emocionales que los racionales.

\section{Estrategias de coaching para la toma de decisiones profesional}

A continuación se presenta una propuesta de estrategias utilizadas en procesos de coaching que pueden resultar efectivas para la toma de decisiones profesional, teniendo en cuenta las tres dimensiones del modelo de toma de decisiones adoptado.

\section{Seis sombreros para pensar}

- Dimensión: cognitiva.

- Objetivos: Llevar a un pensamiento más creativo. Enfocar y enmarcar con claridad el pensamiento. Mejorar la comunicación y así la toma de decisiones.

- Descripción:

Edward de Bono (2005) propone un método sencillo pero efectivo para mejorar nuestra manera de pensar y evitar ser confusos e ineficaces. Si nos "ponemos" un sombrero, podemos centrar y enfocar el pensamiento. Si "cambiamos" de sombrero, podemos redirigir nuestro pensamiento. Si nuestro pensamiento se precisa, nuestra argumentación resulta más centrada y productiva. Para esta dinámica, valoraremos nuestra decisión pasando por cada uno de estos "sombreros" y respondiendo con detalle a las siguientes preguntas: 
- Blanco (objetividad): hechos, cifras, información objetiva. ¿Qué elementos objetivos tengo? ¿Hasta qué punto son reales o probables? ¿Qué está pasando? ¿Cuál es la situación sobre la cual debo decidir?

- Rojo (subjetividad y emoción): emociones y sensaciones, sentimientos que despiertan las distintas opciones . ¿Qué intuición tengo? ¿Qué emociones me despierta cada opción? ¿Cómo me siento ante esta situación?

- Negro (abogado del diablo): lógico, negativo. ¿Parto de la buena base? ¿Es la única posible? ¿Qué variable importante me dejo por analizar? ¿Qué es lo peor que podría pasar ante cada una de las opciones posibles?

- Amarillo (optimismo y alegría): positivo, constructivo, lo que más me ilusiona, lo más interesante. ¿Qué es lo más positivo de todo? ¿Qué es lo que me hace más ilusión? ¿Qué pondré de mi parte para que todo vaya bien? ¿Qué es lo mejor que podría pasar ante cada una de las opciones posibles? ¿Qué intentaría si supiera que no voy a fracasar?

- Verde (esperanza y novedad): creatividad, ideas nuevas. ¿Qué información nueva puedo aportar? ¿Desde qué posición nueva puedo mirar la decisión a tomar? ¿Qué más puedo hacer?

- Azul (coherencia y planificación): control de los demás sombreros, pasos para pensar, planificación del futuro y búsqueda de coherencia e incoherencia. ¿Qué necesito hacer a partir de ahora? ¿Hasta qué punto esta decisión es coherente o no conmigo mismo? ¿Con qué de mi mismo es coherente y con qué de mí y de mi entorno es incoherente? ¿Qué decido finalmente?

\section{Escultura humana}

- Dimensión: emocional.

- Objetivo: "Esculpir" visualmente la actitud que tomamos en el momento de tomar decisiones. 
- Descripción:

Para esta dinámica necesitamos la ayuda de tres personas: el representante del coachee, el facilitador y el bloqueador. Como punto de partida, el/la coachee escenifica corporalmente, junto con un globo que representa el objetivo a conseguir, cómo se posiciona delante una decisión a tomar. El representante del coachee sustituye a la escultura para que el coachee pueda ver la escultura desde fuera. El coachee, desde fuera de la escultura, colocará al facilitador y al bloqueador. El facilitador representa una figura (persona, actitud, creencia, fortaleza, comportamiento, emoción...) que le ayuda en el proceso de decidir. El bloqueador (persona, actitud, creencia, limitación, comportamiento, emoción...) representa una figura que le está dificultando el proceso de decidir.

En todo este proceso de diseño de la escultura el coach da las órdenes de qué hacer en cada momento. Como trabajo profundo, una vez la escena ha quedado definida, el coach pedirá feedback o información al:

- Coachee, respecto a lo que está viendo

- Representante del coachee, respecto a cómo se siente dentro de esta escultura y qué necesitaría hacer

- Facilitador, respecto a cómo se siente dentro de esta escultura y qué necesitaría hacer

- Bloqueador, respecto a cómo se siente dentro de esta escultura, y qué necesita hacer.

El coachee escucha, sin derecho a réplica, las respuestas de los diferentes personajes. Una vez todos los personajes han respondido, empieza un diálogo entre coach y coachee que sea facilitador del trabajo definido. Los otros personajes pueden intervenir en el transcurso del diálogo como co-coaches.

\section{¡Contrólate!}

- Dimensión: Social

- Objetivos:

- Identificar las conductas agresivas, pasivas y asertivas

- Destacar las ventajas y los inconvenientes de las tres conductas (agresiva, pasiva y asertiva) 
- Comprobar que la conducta asertiva es la más adecuada a la hora de afrontar un conflicto y una toma de decisiones.

- Descripción:

El jefe de personal en una reunión en la gerencia de una empresa expone la situación de despido de tres trabajadores. Un trabajador manifiesta su desacuerdo de una forma agresiva e irreflexiva; un segundo trabajador muestra una conducta pasiva, prefiere que le echen y no armar ningún altercado, se conforma y lo acepta de buen grado y un tercero evidencia una conducta asertiva, intentando razonar con el jefe de personal para así poder llegar a un acuerdo que no perjudique a ambas partes.

A través de un role-playing tres personas del grupo tratan de escenificar, de forma exagerada, las tres conductas (agresiva, pasiva y asertiva). Es decir, la persona agresiva ha de amenazar, usando un tono de voz elevado, no ateniéndose a razones, exagerando la situación, no siendo objetivo, pensando que está en posesión de la verdad; la persona pasiva manifiesta su queja en voz baja, sin convicción, sin insistir, asumiendo de buen grado la decisión del jefe de personal; la persona asertiva pide explicaciones, de forma adecuada, sin elevar el tono de voz, insistiendo en la injusticia que se está cometiendo con él, pero aceptando la decisión de la empresa.

Una vez realizado el role-playing se establece una sesión de discusión con el resto del grupo para analizar:

a) Las conductas y comportamientos verbales de los tres personajes (tipo de expresiones, vocabulario, tono de voz, desprecio, ironías, etc.)

b) Las conductas y comportamientos gestuales de los tres personajes (actitud del cuerpo, mirada, movimiento de los brazos, gesticulación, colocación de los brazos, etc.)

c) Las sensaciones que provocan las tres conductas al resto del grupo (rechazo, simpatía, indiferencia, empatía, temor, vergüenza, miedo, ira, etc.)

d) Los valores que se muestran en las conductas (solidaridad, respeto, egoísmo, prepotencia, sumisión, responsabilidad, independencia, altruismo, relación social, seguridad, etc.)

e) Las ventajas y las desventajas de las tres conductas y puesta común. 


\begin{tabular}{|l|l|l|}
\hline Tipos de conducta & Ventajas & Inconvenientes \\
\hline Agresiva & & \\
\hline Pasiva & & \\
\hline Asertiva & & \\
\hline
\end{tabular}

f) Las ventajas de la conducta asertiva y su relación con una adecuada resolución del conflicto y/o toma de decisión.

\section{A modo de conclusión}

La finalidad de este trabajo ha sido relacionar el coaching con los procesos de toma de decisiones. Entendiendo el coaching no como un entrenamiento, sino como una disciplina que va ayudar a la persona a ser, a hacer y a obtener lo que busca; es decir, a autorregularse y a desarrollarse plenamente. $\mathrm{Y}$ en este proceso la toma de decisiones juega un papel muy relevante.

El proceso de coaching ayuda a la persona a desarrollarse personal y profesionalmente a través de la comunicación y hace que las tomas de decisión sean más adecuadas y efectivas; en definitiva el coaching facilita el afrontamiento de situaciones que preocupan a la persona y, entre ellas está la toma de decisiones profesional.

El proceso de la toma de decisiones es una dimensión fundamental de la orientación profesional o desarrollo de la carrera (Career Development). Para su desarrollo se propone un modelo comprensivo que intenta subsanar las deficiencias de los modelos existentes, que se centran fundamentalmente en la dimensión cognitiva. Este modelo para la toma de decisiones profesional asume no sólo la dimensión cognitiva, sino que incluye las dimensiones emocional y social. Dimensiones que, según estudios recientes referenciados en este estudio (Brown, George-Currant y Smith, 2003; Emmerling y Cherniss, 2003; Gaffner y Hazler, 2002), tienen un gran protagonismo en los procesos decisorios. La operativización del modelo se concreta con una serie de estrategias de coaching para cada una de las dimensiones de dicho modelo. 
Este trabajo sobre el proceso de toma de decisiones profesionales a través del coaching pretende ser una significativa aportación para los profesionales que se encargan o bien tienen responsabilidad en la formación profesional inicial, ocupacional y continua. Estos han de asesorar a los usuarios de la formación en las trayectorias formativas más adecuadas, a través de un proceso de toma de decisiones y mediante la orientación del coaching.

\section{Referencias}

Álvarez González, M. (1991). Los efectos de entrenamiento en el proceso de toma de decisiones a través del modelo "DECIDES”. Revista de Investigación Educativa, 9(18), 53 62.

Álvarez González, M. (1995). Orientación profesional. Barcelona: Cedecs.

Álvarez González, M. (2004). El proceso de toma de decisiones. Aspectos a tener en cuenta. En M. Álvarez González y R. Bisquerra (Coords.) Manual de orientación y tutoría (formato electrónico). Barcelona: Praxis.

Álvarez González, M. (2006). Análisis de los principales programas de orientación profesional. En M. Álvarez González y R. Bisquerra (Coords.) Manual de orientación y tutoría (formato electrónico). Barcelona: Praxis.

Álvarez González, M. y Rodríguez Moreno, M. L. (2006). El proceso de toma de decisiones en la educación secundaria. Un enfoque comprensivo. Revista de Orientación Educacional, 20(38), 13-48.

Benavent, J. A. (2002). Programa de autoayuda para la toma de decisiones al finalizar la ESO (CESOF/06). En M. Álvarez González y R. Bisquerra (Coords.) Manual de orientación y tutoría (formato electrónico). Barcelona: Praxis.

Benavent, J. A. (2006). METODE. Bachillerato: Método para la toma de decisiones al finalizar el Bachillerato. Valencia: Tirant lo Blanch.

Bergeron, L. M. y Romano, J. 1. (1994). The Relationships Among Career Decision- Making Self- Efficacy, Educational Indecision, Vocational Indecision and Gender. Journal of College Student Development, 35, 19- 24.

Blustein, D. L. (1987). Decision- Making Styles and Vocational Maturity. An Alternative Perspective. Journal of Vocational Behavior, 30, 61- 71. 
Bransford, J. D. y Stein, B. S. (1993). Solución ideal de problemas. Guía para mejorar, pensar, aprender y crear. Barcelona: Labor.

Brown, C., George-Curran, R. y Smith, M. L. (2003). The Role of Emotional Intelligence in the Career Commitment and Decision Process. Journal of Career Assessment, 11 (4), 379-392.

Callanad, G. y Greenhaus, J. H. (1990). The career indecision of managers and professionals; development of a scale and test of a model. Journal of Vocational Behavior, 37, 79103.

Corominas, E. (1999). Estilo de decisiones de carácter profesional-ocupacional en estudiantes universitarios. En AAVV. Nuevas realidades educativas, nuevas necesidades metodológicas. Málaga: Actas del Congreso Nacional de AIDIPE.

Coronado, A. (2003). Conoce, compara y elige. Programa de toma de decisiones para $4^{\circ}$ de ESO. Madrid: MAD S.L.

De Bono, E. (2005). Seis sombreros para pensar. Buenos Aires: Granica.

Dilts, R. (2004). Coaching. Herramientas para el cambio. Barcelona: Urano.

Emmerling, R. J. y Cherniss, C. (2003). Emocional Intelligence and the Career Choice Process. Journal of Career Assessment, 11(2), 153-167.

Frago, R. y Álvarez Rojo, V. (2006). ;Tengo que decidirme en la ESO!:Un programa para la elección de estudios y profesiones. Cuaderno de actividades. San sebastián: Erein.

Gaffner, D. C. y Hazler, R. J. (2002). Factors Related to Indecisiveness and Career Indecisión in Undecided College Students. Journal of College Student Development, 43(3), 317326.

Gelatt, H. B. (1962). Decision-Making: A Conceptual Frame Of Reference for Counseling. Journal of Counseling Psychology, 9, 240-245.

Gelatt, H. B. (1977). Deciding. Nueva York: The College Board.

Gómez, B. (1995). La toma de decisiones y la indecisión vocacional. En F. Rivas (Coord.) Manual de asesoramiento y orientación vocacional (págs. 281-305). Madrid: Síntesis.

Harren, V. A. (1979). A model of career decision making for college student. Journal of Vocational Behavior, 14, 119-133.

Hilton, T. L. (1962). Career Decision Making. Journal of Counseling Psychology, 9, 291-298.

Holland, J. L. (1973). Making Vocational Choices: A theory of career. Englewood Cliffs, NJ: Prentice-Hall.

Holland, J. L. (1985). Making Vocational Choices: A theory of vocational personalities and work environments (2nd. Ed.). Englewood Cliffs, NJ: Prentice-Hall. 
Jepsen, D. A. (1974). Vocational decision-making strategy types. Vocational Guidance Quarterly, 23, 17-23.

Johnson, R. (1978). Individual Styles of decision making: A theoretical model for counselling. Personnel \& Guidance Journal, 56, 530-536.

Kraus, L. J. y Hughey, P. D. (1999). The Impact of an Intervention on Career Decision- Making Self- Efficacy and Career Indecision. Professional School Counseling, 2(5), 384390.

Krumboltz, J. D. y Hamel, D. A. (1977). Guidance Career Decision Making Skills. New York: College Entrance Examination Board.

Krumboltz, J. D. (1979). A Social Learning Theory of Career Decision Making. En A. M. Mitchell, G. B. Jones. y J. D. Krumboltz (Eds.). Social Learning and Career Decision Making (págs. 19-50). Cranston: The Carroll Press.

Launer, V. (2007). Coaching. Un camino hacia nuestros éxitos. Madrid: Pirámide.

Ma, P. W. y Yeh, C. J. (2005). Factors Influencing the Career Status of Chinese American Youths. The Career Development Quarterly, 53(4), 337- 347.

Mayer, J. D. y Salovey, P. (1997). What is emotional intelligence?. En P. Salovey y D. Sluyter (Eds.). Emotional development and emotional intelligence: Implications for education (3-14). Nueva York: Basic Books.

Mayer, J. D., Caruso, D. y Salovey, P. (1999). Emotional intelligence meets tradicional standards for an intelligence. Intelligence, 27, 267-298.

Mellers, B. A., Schwartz, A. y Ritov. I. (1999). Emotion-based choice. Journal of Experimental Psychology: General, 128(3), 332-345.

Miedaner, T. (2004). Coaching para el éxito. Barcelona: Urano.

Niles, S. G., Erford, B. T., Hunt, B. y Watts, R. H. (1997). Decision- Making Styles and Career Development in College Students. Journal of College Student Development, 38 (5), 479- 488 .

Osipow, (1983). Teorías del desarrollo de la carrera. México: Trillas.

Pelletier, D. y Cols (1991). La définition de la décision (13-15). Québec: Les Éditions. Septembre.

Rivas, F. (1988). Psicología vocacional: Enfoques del asesoramiento. Madrid: Morata.

Rodríguez Moreno, M. L.; Dorio, I. y Morey, M. (1994). Programa para enseñar a tomar decisiones. Barcelona: Laertes.

Super, D. E. (1977). Un modelo de desarrollo de la vida como carrera. Revista de Psicología General y Aplicada, 147, 663-683. 
Tiedeman, D. V. y O’Hara, R. P. (1963). Career Development: Choice and Adjustment. New York: College Entrance Examination Board.

Whitmore, J. (2003). Coaching. El método para mejorar el rendimiento de las personas. Barcelona: Paidós.

Wolk, L. (2004). Coaching. El arte de soplar brasas. Buenos Aires: Gran Aldea Editores.

Zeus, P. y Skiffington, S. (2004). Coaching práctico en el trabajo. Madrid: Mc Graw Hill. 